IoAnNis K. Argyros (Lawton, OK)

RAMANDEeP BeHL (Jeddah)

S. S. Motsa (Scottsville and Kwaluseni)

\title{
BALL CONVERGENCE FOR A TWO-STEP FOURTH ORDER DERIVATIVE-FREE METHOD FOR NONLINEAR EQUATIONS
}

Abstract. We present a local convergence analysis of a two-step fourth order derivative-free method in order to approximate a locally unique solution of a nonlinear equation in a real or complex space setting. In an earlier study of Peng et al. (2011), the order of convergence of the method was shown using Taylor series expansions and hypotheses on up to the fourth order derivative or even higher of the function involved. However, no derivative appears in the proposed scheme. That restricts the applicability of the scheme. We expand the applicability of the scheme using only hypotheses on the first order derivative of the function involved. We also give computable radii of convergence, error bounds based on Lipschitz constants, and the range of initial guesses that guarantees convergence of the methods. Numerical examples where earlier studies do not apply but our results do are also given.

1. Introduction. In this study we are concerned with the problem of approximating a locally unique solution $x^{*}$ of the equation of the form

$$
F(x)=0,
$$

where $F$ is a twice Fréchet differentiable function defined on a subset $D$ of $S$ $(S=\mathbb{R}$ or $S=\mathbb{C})$ with values in $S$.

Lack or intractability of analytic solutions often forces researchers to resort to iterative methods. While using these methods, researchers face the

2010 Mathematics Subject Classification: 65D10, 65D99.

Key words and phrases: two-step method with memory, local convergence, convergence order.

Received 18 January 2017; revised 2 June 2017.

Published online 15 April 2019. 
problems of slow convergence, non-convergence, divergence, inefficiency or failure (for details see Traub [14 and Petkovic et al. [12]). The convergence analysis of iterative methods is usually divided into two categories: semilocal and local convergence analysis. The semi-local analysis is, relying on information around an initial point, to give criteria ensuring the convergence of iteration procedures. An important problem in the study of iterative procedures is the convergence domain. Therefore, it is crucial to estimate the radius of convergence of the method applied.

We conduct the local convergence analysis of the two-step fourth order method defined for $n=0,1,2, \ldots$ by

$$
\begin{aligned}
y_{n} & =x_{n}-\frac{F\left(x_{n}\right)}{G\left(x_{n}\right)} \\
x_{n+1} & =x_{n}-\frac{F\left(x_{n}\right)}{G\left(x_{n}\right)}\left[1+\frac{F\left(y_{n}\right)}{F\left(x_{n}\right)}+\left(1+\frac{1}{1-\alpha_{n} G\left(x_{n}\right)}\right)\left(\frac{F\left(y_{n}\right)}{F\left(x_{n}\right)}\right)^{2}\right],
\end{aligned}
$$

where $x_{0}$ is a initial point, $\alpha_{n} \in S$ and

$$
\begin{aligned}
G\left(x_{n}\right) & =\frac{F\left(x_{n}\right)-F\left(x_{n}-\alpha_{n} F\left(x_{n}\right)\right)}{\alpha_{n} F\left(x_{n}\right)}, \\
& =\frac{\int_{0}^{1} F^{\prime}\left(x_{n}-\alpha_{n} F\left(x_{n}\right)+\theta \alpha_{n} F\left(x_{n}\right)\right) \alpha_{n} F\left(x_{n}\right) d \theta}{\alpha_{n} F\left(x_{n}\right)} \\
& =\int_{0}^{1} F^{\prime}\left(x_{n}-\alpha_{n} F\left(x_{n}\right)+\theta \alpha_{n} F\left(x_{n}\right)\right) d \theta:=A_{n} .
\end{aligned}
$$

The sequence $\left\{x_{n}\right\}$ is adaptively determined. The local convergence of method (1.2) was studied in 11] using hypotheses reaching up to the fourth derivative of $F$ when $S=\mathbb{R}$. Such hypotheses limit the applicability of the method. As a motivational example, for $S=\mathbb{R}$ define a function $F$ on $D=[-1 / \pi, 2 / \pi]$ by

$$
F(x)= \begin{cases}x^{3} \log \left(\pi^{2} x^{2}\right)+x^{5} \sin \left(\frac{1}{x}\right), & x \neq 0, \\ 0, & x=0 .\end{cases}
$$

Then

$$
\begin{aligned}
F^{\prime}(x) & =2 x^{2}-x^{3} \cos \left(\frac{1}{x}\right)+3 x^{2} \log \left(\pi^{2} x^{2}\right)+5 x^{4} \sin \left(\frac{1}{x}\right), \\
F^{\prime \prime}(x) & =-8 x^{2} \cos \left(\frac{1}{x}\right)+2 x\left(5+3 \log \left(\pi^{2} x^{2}\right)\right)+x\left(20 x^{2}-1\right) \sin \left(\frac{1}{x}\right), \\
F^{\prime \prime \prime}(x) & =\frac{1}{x}\left[\left(1-36 x^{2}\right) \cos \left(\frac{1}{x}\right)+x\left(22+6 \log \left(\pi^{2} x^{2}\right)+\left(60 x^{2}-9\right) \sin \left(\frac{1}{x}\right)\right)\right] .
\end{aligned}
$$

One can easily find that $F^{\prime \prime \prime}(x)$ is unbounded on $D$ at the point $x=0$. Hence, the results in [11] cannot apply to show the convergence of method (1.2) or its special cases requiring hypotheses on the fourth derivative of $F$ or higher. 
Notice that there are a plethora of iterative methods for approximating solutions of nonlinear equations $1,10,12,14$. These results show that the initial guess should be close to the required root for the convergence of the corresponding method. But how close? The local results give no information on the radius of the convergence ball for the corresponding method.

Peng et al. [11] provided several reasons why the investigation of method (1.2) is worthwhile. We repeat some of them here and refer the reader to 11] for the rest. For example, method (1.2) is derivative-free and of high convergence order, in contrast to other methods in the literature of the same order but using derivatives which may be expensive or impossible to compute or do not exist. The study of local convergence based on Lipschitz constants is important because it shows the degree of difficulty in choosing initial guesses. In studies using Taylor expansions such as 11] the choice of the initial guess $x_{0}$ is a "shot in the dark". Clearly, our technique can be applied to other iterative methods with similar advantages.

In the present study we expand the applicability of method $(1.2)$ using only hypotheses on up to the first order derivative of $F$. We also give computable radii of convergence and error bounds based on Lipschitz constants. We further present the range of initial guesses $x_{0}$ that tells us how close the initial guess should be for method (1.2) to converge. This problem was not addressed in [11. The advantages of our approach are similar to the ones already mentioned for method 1.2 .

2. Local convergence. In this section, we shall define some scalar functions and parameters in order to analyze the local convergence of method (1.2).

Let $L_{0}, L>0, M \geq 1, M_{0}>0$ and $\alpha>0$ be given parameters. Let us define functions $p, g_{1}$ and $h_{1}$ on $\left[0,1 / L_{0}\right)$ by

$$
\begin{aligned}
p(t) & =L_{0}\left(1+\frac{1}{2} \alpha M\right) t, \\
g_{1}(t) & =\frac{L}{2\left(1-L_{0} t\right)}\left(1+\frac{\alpha M_{0} M}{1-p(t)}\right) t, \\
h_{1}(t) & =g_{1}(t)-1,
\end{aligned}
$$

and a parameter $r_{p}$ by

$$
r_{p}=\frac{1}{\left(1+\frac{1}{2} \alpha M\right) L_{0}} .
$$

We have $h_{1}(0)=-1$, and $h_{1}(t), h_{p}(t) \rightarrow \infty$ as $t \rightarrow r_{p}^{-}$. By the intermediate value theorem, $h_{1}$ has zeros in $\left(0, r_{p}\right)$. Let $r_{1}$ be the smallest such zero. Suppose that

$$
\alpha M_{0}<1
$$


Moreover, define functions $g_{2}$ and $h_{2}$ on $\left[0, r_{1}\right)$ by

$$
\begin{aligned}
& g_{2}(t)=\left(1+\frac{M}{1-p(t)}+\frac{M^{3}}{1-p(t)}\left(1+\frac{1}{1-\alpha M_{0}}\right) g_{1}(t)\right) g_{1}(t), \\
& h_{2}(t)=g_{2}(t)-1 .
\end{aligned}
$$

Then $h_{2}(0)=-1<0$ and

$$
h_{2}\left(r_{1}\right)=\frac{M}{1-p\left(r_{1}\right)}+\frac{M^{3}}{1-p\left(r_{1}\right)}\left(1+\frac{1}{1-\alpha M_{0}}\right)>0,
$$

since $g_{1}\left(r_{1}\right)=1,1-|\alpha| M>0, r_{1}<r_{p}$ and $1-p\left(r_{1}\right)>0$. Denote by $r$ the smallest zero of $h_{2}$ in $\left[0, r_{1}\right)$. Then for each $t \in[0, r)$,

$$
\begin{aligned}
& 0 \leq p(t)<1, \\
& 0 \leq g_{1}(t)<1, \\
& 0 \leq g_{2}(t)<1,
\end{aligned}
$$

Let $U(\gamma, \rho)$ and $\bar{U}(\gamma, \rho)$ be respectively the open and closed balls in $S$ with center $\gamma \in S$ and radius $\rho>0$. Next, we present the local convergence analysis of method (1.2) using the above notations.

Theorem 2.1. Let $F: S \supset D \rightarrow S$ be a differentiable function. Suppose that there exist $x^{*} \in D$ and $L_{0}>0$ such that

$$
F\left(x^{*}\right)=0, \quad F^{\prime}\left(x^{*}\right) \neq 0,
$$

and for each $x \in D$,

$$
\left|F\left(x^{*}\right)^{-1}\left(F^{\prime}(x)-F^{\prime}\left(x^{*}\right)\right)\right| \leq L_{0}\left|x-x^{*}\right| .
$$

Moreover, suppose that there exist $L>0, M \geq 1, M_{0} \in[1,1 / \alpha), \alpha>0$ and a sequence $\left\{\alpha_{n}\right\} \subset S$ such that for each $x, y \in D \cap U\left(x^{*}, 1 / L_{0}\right)$,

$$
\begin{aligned}
& \left|\alpha_{n}\right| \leq \alpha, \\
& \left|F^{\prime}\left(x^{*}\right)^{-1}\left(F^{\prime}(x)-F^{\prime}(y)\right)\right| \leq L|x-y|, \\
& \left|F^{\prime}\left(x^{*}\right)^{-1} F^{\prime}(x)\right| \leq M, \quad\left|F^{\prime}(x)\right| \leq M_{0}, \\
& \bar{U}\left(x^{*},\left(1+\alpha M_{0}\right) r\right) \subseteq D,
\end{aligned}
$$

where $r$ has been defined previously. Then the sequence $\left\{x_{n}\right\}$ generated for $x_{0} \in U\left(x^{*}, r\right)-\left\{x^{*}\right\}$ by method 1.2 is well defined, remains in $U\left(x^{*}, r\right)$ for each $n=0,1,2, \ldots$ and converges to $x^{*}$. Moreover,

$$
\begin{aligned}
& \left|y_{n}-x^{*}\right| \leq g_{1}\left(\left|x_{n}-x^{*}\right|\right)\left|x_{n}-x^{*}\right|<\left|x_{n}-x^{*}\right|<r, \\
& \left|x_{n+1}-x^{*}\right| \leq g_{2}\left(\left|x_{n}-x^{*}\right|\right)\left|x_{n}-x^{*}\right|<\left|x_{n}-x^{*}\right|,
\end{aligned}
$$

where the " $g$ " functions have been defined previously. Furthermore, for $T \in$ $\left[r, 2 / L_{0}\right)$, the limit point $x^{*}$ is the only solution of the equation $F(x)=0$ in $D_{0}:=\bar{U}\left(x^{*}, T\right) \cap D$. 
Proof. We shall show (2.11) and (2.12) by induction. By the hypothesis $x_{0} \in U\left(x^{*}, r\right)-\left\{x^{*}\right\}$, the definition of $r$ and (2.6), we have

$$
\left|F^{\prime}\left(x^{*}\right)^{-1}\left(F^{\prime}\left(x_{0}\right)-F^{\prime}\left(x^{*}\right)\right)\right| \leq L\left|x_{0}-x^{*}\right|<L_{0} r<1 .
$$

It follows from (2.13) and the Banach Lemma on invertible functions [4, 13 that $F^{\prime}\left(x_{0}\right) \neq 0$ and

$$
\left|F^{\prime}\left(x^{*}\right)^{-1}\left(F^{\prime}\left(x_{0}\right)-F^{\prime}\left(x^{*}\right)\right)\right| \leq \frac{1}{1-L_{0}\left|x_{0}-x^{*}\right|}<\frac{1}{1-L_{0} r} .
$$

By 2.5 we can write

$$
F\left(x_{0}\right)=F\left(x_{0}\right)-F\left(x^{*}\right)=\int_{0}^{1} F^{\prime}\left(x^{*}+\theta\left(x_{0}-x^{*}\right)\right)\left(x_{0}-x^{*}\right) d \theta .
$$

Notice that $\left|x^{*}+\theta\left(x_{0}-x^{*}\right)-x^{*}\right|=\theta\left|x_{0}-x^{*}\right|<r$. Hence, by (2.9) and (2.15),

$$
\left|F^{\prime}\left(x^{*}\right)^{-1} F\left(x_{0}\right)\right| \leq M\left|x_{0}-x^{*}\right| .
$$

We must show that $A_{0} \neq 0$. Notice that

$$
\left|x_{0}-\alpha_{0} F\left(x_{0}\right)-x^{*}\right| \leq\left|x_{0}-x^{*}\right|+|\alpha| M\left|x_{0}-x^{*}\right|<(1+|\alpha| M) r
$$

and

$$
\begin{aligned}
\left|x_{0}+\alpha_{0} F\left(x_{0}\right)(\theta-1)-x^{*}\right| & \leq\left|x_{0}-x^{*}\right|+(1-\theta) M_{0} \alpha\left|x_{0}-x^{*}\right| \\
& <\left(1+\alpha M_{0}\right) r,
\end{aligned}
$$

so $x_{0}-\alpha_{0} F\left(x_{0}\right), x_{0}+\alpha_{0} F\left(x_{0}\right)(\theta-1) \in U\left(x^{*},\left(1+\alpha M_{0}\right) r\right)$. Then, using (2.2), the definition of $r$ and (2.6), we get in turn

$$
\begin{aligned}
\left|F^{\prime}\left(x^{*}\right)^{-1}\left(A_{0}-F^{\prime}\left(x^{*}\right)\right)\right| & \leq \int_{0}^{1}\left|x_{0}-x^{*}+(\theta-1) \alpha_{0} F\left(x_{0}\right)\right| d \theta \\
& \leq L_{0}\left(1+\alpha \int_{0}^{1}(\theta-1) d \theta M_{0}\left|x_{0}-x^{*}\right|\right) \\
& \leq L_{0}\left(1+M_{0} \alpha / 2\right)\left|x_{0}-x^{*}\right| \\
& =p\left(\left|x_{0}-x^{*}\right|\right)<p\left(r_{p}\right)=1 .
\end{aligned}
$$

Then, by (2.19), we have $A_{0} \neq 0$ and

$$
\left|A_{0}^{-1} F^{\prime}\left(x^{*}\right)\right| \leq \frac{1}{1-p\left(\left|x_{0}-x^{*}\right|\right)}<\frac{1}{1-p(r)}
$$

and $y_{0}$ is well defined by the first substep of method 1.2 for $n=0$. By (2.8), 2.9) and (2.16), we also get

$$
\left|F^{\prime}\left(x^{*}\right)^{-1}\left(A_{0}-F^{\prime}\left(x_{0}\right)\right)\right| \leq L \int_{0}^{1}(1-\theta) d \theta M_{0}\left|x_{n}-x^{*}\right| .
$$


Then, using the first substep of method $(1.2)$ for $n=0,(2.3),(2.8),(2.9)$, (2.14, 2.16), 2.20 and (2.21) we get in turn

(2.22) $\quad\left|y_{0}-x^{*}\right|$

$$
\begin{aligned}
& \leq\left|F^{\prime}\left(x_{0}\right)^{-1} F^{\prime}\left(x^{*}\right)\right|\left|\int_{0}^{1} F^{\prime}\left(x^{*}\right)^{-1}\left[F^{\prime}\left(x^{*}+\theta\left(x_{0}-x^{*}\right)\right)-F^{\prime}\left(x_{0}\right)\right]\left(x_{0}-x^{*}\right) d \theta\right| \\
& +\left|F^{\prime}\left(x_{0}\right)^{-1} F^{\prime}\left(x^{*}\right)\right|\left|F^{\prime}\left(x^{*}\right)^{-1}\left(A_{0}-F^{\prime}\left(x_{0}\right)\right)\right|\left|A_{0}^{-1} F^{\prime}\left(x^{*}\right)\right|\left|F^{\prime}\left(x^{*}\right)^{-1} F\left(x_{0}\right)\right| \\
& \leq \frac{L\left|x_{0}-x^{*}\right|^{2}}{2\left(1-L_{0}\left|x_{0}-x^{*}\right|\right)}+\frac{L M_{0} M \alpha\left|x_{0}-x^{*}\right|^{2}}{2\left(1-L_{0}\left|x_{0}-x^{*}\right|\right)\left(1-p\left(\left|x_{0}-x^{*}\right|\right)\right)} \\
& =g_{1}\left(\left|x_{0}-x^{*}\right|\right)\left|x_{0}-x^{*}\right|<\left|x_{0}-x^{*}\right|<r,
\end{aligned}
$$

which shows 2.11) for $n=0$ and $y_{0} \in U\left(x^{*}, r\right)$. We also see that 2.16) holds for $y_{0}=x_{0}$, since $y_{0} \in U\left(x^{*}, r\right)$. We must show that $F\left(x_{0}\right) \neq 0$ and $1-\alpha_{0} A_{0} \neq 0$. From (2.5), 2.6 and $x_{0} \neq x^{*}$ we get

$$
\begin{aligned}
& \left|\left(F^{\prime}\left(x^{*}\right)\left(x_{0}-x^{*}\right)\right)^{-1}\left[F\left(x_{0}\right)-F\left(x^{*}\right)-F^{\prime}\left(x^{*}\right)\left(x_{0}-x^{*}\right)\right]\right| \\
& \quad \leq\left|x_{0}-x^{*}\right|^{-1} \frac{L_{0}}{2}\left|x_{0}-x^{*}\right|^{2}=\frac{L_{0}}{2}\left|x_{0}-x^{*}\right|<\frac{L_{0}}{2} r<1 .
\end{aligned}
$$

By 2.23 , we have $F\left(x_{0}\right) \neq 0$ and

$$
\left|F\left(x_{0}\right)^{-1} F^{\prime}\left(x^{*}\right)\right| \leq \frac{1}{\left|x_{0}-x^{*}\right|\left(1-\frac{L_{0}}{2}\left|x_{0}-x^{*}\right|\right)} .
$$

By (2.7) and 2.9 with $x_{0}-\alpha_{0} F\left(x_{0}\right)+\theta \alpha_{0} F\left(x_{0}\right)$ replacing $x$ we also get

$$
\left|\alpha_{0} A_{0}\right| \leq\left|\alpha_{0}\right|\left|A_{0}\right| \leq \alpha M_{0}<1 .
$$

That is $A_{0} \neq 0, x_{1}$ is well defined by the second substep of method 1.2 for $n=0$ and

$$
\left|\left(I-\alpha_{0} A_{0}\right)^{-1}\right| \leq \frac{1}{1-\alpha M_{0}}
$$

By the last substep of method 1.2 for $n=0$ we can write

$$
x_{1}=y_{0}-\frac{F\left(y_{0}\right)}{A_{0}}-\frac{F\left(x_{0}\right)}{A_{0}}\left(1+\frac{1}{1-\alpha_{0} A_{0}}\right)\left(\frac{F\left(y_{0}\right)}{F\left(x_{0}\right)}\right)^{2} \text {. }
$$

Then, in view of (2.4), the definition of $r, 2.14,2.16,2.20,2.24,2.26$ 
and 2.27), we obtain

$$
\begin{aligned}
\leq & \left|y_{0}-x^{*}\right|+\left|A_{0}^{-1} F^{\prime}\left(x^{*}\right)\right|\left|F^{\prime}\left(x^{*}\right)^{-1} F\left(y_{0}\right)\right| \\
& +\left|A_{0}^{-1} F^{\prime}\left(x^{*}\right)\right|\left|F^{\prime}\left(x^{*}\right)^{-1} F\left(x_{0}\right)\right|\left[1+\left|\left(1-\alpha_{0} A_{0}\right)^{-1}\right|\right] \\
& \times\left.\left|F\left(x_{0}\right)^{-1} F^{\prime}\left(x^{*}\right)\right|^{2}|| F^{\prime}\left(x^{*}\right)^{-1} F\left(y_{0}\right)\right|^{2} \\
\leq & \left|y_{0}-x^{*}\right|+\frac{M\left(\left|y_{0}-x^{*}\right|+\left|x_{0}-x^{*}\right|\right)}{1-p\left(\left|x_{0}-x^{*}\right|\right)}\left(1+\frac{1}{1-\alpha M}\right) \\
& \times \frac{M^{2}\left|y_{0}-x^{*}\right|^{2}}{\left|x_{0}-x^{*}\right|^{2}\left(1-\frac{L_{0}}{2}\left|x_{0}-x^{*}\right|\right)^{2}} \\
\leq & {\left[1+\frac{M^{3}}{1-p\left(\left|x_{0}-x^{*}\right|\right)}+\frac{1}{1-p\left(\left|x_{0}-x^{*}\right|\right)}\left(1+\frac{1}{1-\alpha M}\right) g_{1}\left(\left|x_{0}-x^{*}\right|\right)\right] }
\end{aligned}
$$

which shows 2.12 for $n=0$ and $x_{1} \in U\left(x^{*}, r\right)$. By simply replacing $x_{0}, y_{0}, x_{1}$ by $x_{k}, y_{k}, x_{k+1}$ in the preceding estimates we arrive at (2.11) and (2.12). Then notice that $\left|x_{k+1}-x^{*}\right| \leq c\left|x_{k}-x^{*}\right|<r, c=g_{2}(r) \in[0,1)$. Hence, $\lim _{k \rightarrow \infty} x_{k}=x^{*}$ and $x_{k+1} \in U\left(x^{*}, r\right)$. Finally, to show uniqueness, let $y^{*} \in \bar{U}\left(x^{*}, T\right)$ be such that $F\left(y^{*}\right)=0$. Set $Q=\int_{0}^{1} F^{\prime}\left(x^{*}+\theta\left(y^{*}-x^{*}\right)\right) d \theta$. Then, using 2.12, we get

$$
\left|F^{\prime}\left(x^{*}\right)^{-1}\left(Q-F^{\prime}\left(x^{*}\right)\right)\right| \leq L_{0} \int_{0}^{1} \theta\left|x^{*}-y^{*}\right| d \theta=\frac{L_{0}}{2} T<1 .
$$

Hence, $Q^{-1} \in L(Y, X)$. Then, in view of the identity $F\left(y^{*}\right)-F\left(x^{*}\right)=$ $Q\left(y^{*}-x^{*}\right)$, we conclude that $x^{*}=y^{*}$.

REMARK 2.2. (a) In view of (2.6) and the estimate

$$
\begin{aligned}
\left|F^{\prime}\left(x^{*}\right)^{-1} F^{\prime}(x)\right| & =\left|F^{\prime}\left(x^{*}\right)^{-1}\left(F^{\prime}(x)-F^{\prime}\left(x^{*}\right)\right)+I\right| \\
& \leq 1+\left|F^{\prime}\left(x^{*}\right)^{-1}\left(F^{\prime}(x)-F^{\prime}\left(x^{*}\right)\right)\right| \leq 1+L_{0}\left|x_{0}-x^{*}\right|
\end{aligned}
$$

the first condition in 2.9 can be dropped and $M$ can be replaced by

$$
M=M(t)=1+L_{0} t
$$

or $M=2$, since $t \in\left[0,1 / L_{0}\right)$.

(b) The results obtained here can be used for operators $F$ satisfying the autonomous differential equation [4,5] of the form

$$
F^{\prime}(x)=P(F(x))
$$

where $P: S \rightarrow S$ is a known continuous operator. Since $F^{\prime}\left(x^{*}\right)=P\left(F\left(x^{*}\right)\right)$ $=P(0)$, we can apply the results without actually knowing the solution $x^{*}$. 
As an example let $F(x)=e^{x}+1$. Then we can choose $P(x)=x-1$. Notice that for these choices of $F$ and $P$ equation 2.30 is satisfied. Then, for example (2.8) can be written as

$$
\left|P(0)^{-1}(P(F(x)-P(F(y))))\right| \leq L|x-y|,
$$

which is independent of $x^{*}$ (see also Example 3.1 for the value of $L$ ).

(c) The radius $r_{A}=\frac{2}{L_{0}+L}$ was shown by us in 4,5 to be the convergence radius for Newton's method under conditions (2.5), (2.6) and (2.8). The radius $r_{A}$ is at least as large as the convergence ball given by Rheinboldt 13 and Traub [14],

$$
r_{R}=\frac{2}{3 L}
$$

Notice that for $L_{0}<L$,

$$
r_{R}<r_{A}
$$

Moreover,

$$
\frac{r_{R}}{r_{A}} \rightarrow \frac{1}{3} \quad \text { as } \quad \frac{L_{0}}{L} \rightarrow 0
$$

Hence, $r_{A}$ is at most three times larger than $r_{R}$. In the numerical examples we compare $r$ to $r_{A}$ and $r_{R}$.

3. Numerical example and applications. In order to demonstrate the convergence behavior of the scheme proposed in [11] and to check the validity and effectiveness of the theoretical results which we have proposed in Section 2, we choose a variety of nonlinear equations, including our motivational example.

In Tables 1 3, we display the test functions considered with the corresponding initial approximations, radius of convergence and minimum number of iterations $(n)$ to get the desired accuracy. The initial approximation $x_{0}$ is selected within the convergence domain. All the values of parameters have been calculated up to more than 100 significant digits, but in the tables only five significant digits are displayed.

In order to verify the theoretical order of convergence, we use the following formulas to calculate the computational order of convergence (COC) 7

$$
\rho=\frac{\ln \frac{\left|x_{n+2}-x^{*}\right|}{\left|x_{n+1}-x^{*}\right|}}{\ln \frac{\left|x_{n+1}-x^{*}\right|}{\left|x_{n}-x^{*}\right|}} \quad \text { for } n=0,1, \ldots
$$

or the approximate computational order of convergence (ACOC) 7

$$
\rho^{*}=\frac{\ln \frac{\left|x_{n+2}-x_{n+1}\right|}{\left|x_{n+1}-x_{n}\right|}}{\ln \frac{\left|x_{n+1}-x_{n}\right|}{\left|x_{n}-x_{n-1}\right|}} \quad \text { for } n=1,2, \ldots
$$


Finding the order of convergence in this way, we can avoid the error bounds involving estimates of higher than first order Fréchet derivatives. All the numerical experiments have been done in Mathematica (Version 9) with multiple precision arithmetic, which minimize round-off errors. We use $\epsilon=$ $10^{-100}$ as a tolerance error. The following stopping criteria are used for computer programs: (i) $\left|x_{n+1}-x_{n}\right|<\epsilon$ and (ii) $\left|f\left(x_{n+1}\right)\right|<\epsilon$.

Example 3.1. Let $S=\mathbb{R}$ and $D=\bar{U}(0,1)$. Define $F$ on $D$ by

$$
F(x)=e^{x}-1 .
$$

Then

$$
F^{\prime}(x)=e^{x} .
$$

Notice that $x^{*}=0, L_{0}=e-1, L=1.789572397$ and $M=M_{0}=2$. We give different radii of convergence, $\mathrm{COC} \rho$ and $n$ in Table 1 .

Table 1. Computable radii of convergence

\begin{tabular}{cccccccccc}
\hline$\alpha$ & $r_{p}$ & $r_{A}$ & $r_{R}$ & $r_{1}$ & $r_{2}$ & $r$ & $x_{0}$ & $n$ & $\rho$ \\
\hline$\frac{1}{3}$ & 0.43648 & 0.57015 & 0.37253 & 0.20546 & 0.051503 & 0.051503 & 0.05 & 5 & 4.0000 \\
$\frac{1}{4}$ & 0.46558 & 0.57015 & 0.37523 & 0.22873 & 0.064057 & 0.064057 & 0.06 & 5 & 4.0000 \\
$\frac{1}{5}$ & 0.48498 & 0.57015 & 0.37528 & 0.24597 & 0.072475 & 0.072475 & 0.07 & 5 & 4.0000 \\
\hline
\end{tabular}

Example 3.2. Let $S=\mathbb{R}$ and $D=\bar{U}(0,1)$. Define $F$ on $D$ by

$$
F(x)=\sin x .
$$

Then

$$
F^{\prime}(x)=\cos x .
$$

Notice that $x^{*}=0$ and $L_{0}=L=M=M_{0}=1$. We give different radii of convergence, $\operatorname{COC} \rho$ and $n$ in Table 2 .

Table 2. Computable radii of convergence

\begin{tabular}{cccccccccc}
\hline$\alpha$ & $r_{p}$ & $r_{A}$ & $r_{R}$ & $r_{1}$ & $r_{2}$ & $r$ & $x_{0}$ & $n$ & $\rho$ \\
\hline$\frac{1}{2}$ & 0.80000 & 1.0000 & 0.066667 & 0.47340 & 0.24305 & 0.24305 & 0.23 & 4 & 5.0000 \\
$\frac{1}{3}$ & 0.85714 & 1.0000 & 0.66667 & 0.51987 & 0.27579 & 0.27579 & 0.26 & 4 & 5.0000 \\
$\frac{1}{4}$ & 0.88889 & 1.0000 & 0.66667 & 0.54774 & 0.29419 & 0.29419 & 0.28 & 4 & 5.0000 \\
\hline
\end{tabular}

EXAMPLE 3.3. Returning to the motivational example of the introduction, we have $L=L_{0}=\frac{2}{2 \pi+1}\left(80+16 \pi+(11+12 \log 2) \pi^{2}\right), M=2$, $M_{0}=8.5145$ and our required zero is $x^{*}=1 / \pi$. We list different radii of convergence, COC $\rho$ and $n$ in Table 3 . 
Table 3. Computable radii of convergence

\begin{tabular}{cccccccccc}
\hline$\alpha$ & $r_{p}$ & $r_{A}$ & $r_{R}$ & $r_{1}$ & $r_{2}$ & $r$ & $x_{0}$ & $n$ & $\rho$ \\
\hline$\frac{1}{9}$ & 0.010212 & 0.011347 & 0.0075648 & 0.0037799 & 0.00050153 & 0.00050153 & 0.318 & 4 & 4.0000 \\
$\frac{1}{10}$ & 0.011347 & 0.011347 & 0.075648 & 0.0039249 & 0.00075431 & 0.00075431 & 0.319 & 4 & 4.0000 \\
\hline
\end{tabular}

REMARK 3.4. It is worth noticing that the radius $\rho$ is smaller than the radii of Newton's method. This observation is expected, since method 1.2 is of order four, whereas Newton's method is only of order two. In general, as the convergence order increases, the radius of convergence decreases. This is true for all the preceding numerical examples.

4. Conclusions. Numerous iterative methods are available in the literature where researchers state that the initial approximation should be close to the required root to guarantee convergence of the proposed schemes. But most of the time they do not specify how clos that should be. In this study, we give a computable radius of convergence and error bound which we can calculate by using Lipschitz conditions. Further, we also reduce the hypotheses from fourth order derivative of the function involved to only the first order derivative. It is worth noticing that method $(1.2)$ does not change if we use the conditions of Theorem 2.1 instead of the stronger conditions proposed by Peng et al. [11]. Moreover, to obtain the error bounds in practice and order of convergence, we can use the computational order of convergence. In this way, we can avoid the bounds involving estimates higher than the first Fréchet derivative. Thus, our study expands the applicability of the proposed methods. We have also given the idea of how to calculate the computable radius of convergence and the error bounds of the scheme.

\section{References}

[1] S. Amat, S. Busquier and S. Plaza, Dynamics of the King and Jarratt iterations, Aequationes Math. 69 (2005), 212-223.

[2] S. Amat, S. Busquier and S. Plaza, Chaotic dynamics of a third-order Newton-type method, J. Math. Anal. Appl. 366 (2010), 24-32.

[3] S. Amat, M. A. Hernández and N. Romero, A modified Chebyshev's iterative method with at least sixth order of convergence, Appl. Math. Comput. 206 (2008), 164-174.

[4] I. K. Argyros, Convergence and Application of Newton-type Iterations, Springer, 2008.

[5] I. K. Argyros and S. Hilout, Numerical Methods in Nonlinear Analysis, World Sci., 2013.

[6] R. Behl and S. S. Motsa, Geometric construction of eighth-order optimal families of Ostrowski's method, Sci. World J. 2015, art. 614612, 11 pp.

[7] J. A. Ezquerro and M. A. Hernández, New iterations of R-order four with reduced computational cost, BIT Numer. Math. 49 (2009), 325-342. 
[8] V. Kanwar, R. Behl and K. K. Sharma, Simply constructed family of a Ostrowski's method with optimal order of convergence, Computers Math. Appl. 62 (2011), 40214027.

[9] Á. A. Magreñán, Different anomalies in a Jarratt family of iterative root-finding methods, Appl. Math. Comput. 233 (2014), 29-38.

[10] Á. A. Magreñán, A new tool to study real dynamics: The convergence plane, Appl. Math. Comput. 248 (2014), 215-224.

[11] Y. Peng, H. Feng, Q. Li and X. Zhang, A fourth-order derivative free algorithm for nonlinear equations, J. Comput. Appl. Math. 235 (2011), 2551-2559.

[12] M. S. Petković, B. Neta, L. Petković and J. Džunić, Multipoint Methods for Solving Nonlinear Equations, Elsevier, 2013.

[13] W. C. Rheinboldt, An adaptive continuation process for solving systems of nonlinear equations, in: Mathematical Models and Numerical Methods (Warszawa, 1975), Banach Center Publ. 3, PWN, Warszawa, 1978, 129-142.

[14] J. F. Traub, Iterative Methods for the Solution of Equations, Prentice-Hall, Englewood Cliffs, NJ, 1964.

Ioannis K. Argyros

Department of Mathematics Sciences

Cameron University

Lawton, OK 73505, U.S.A.

E-mail: iargyros@cameron.edu

S. S. Motsa

School of Mathematics, Statistics and Computer Science

University of KwaZulu-Natal

Private Bag X01

Scottsville 3209, Pietermaritzburg, South Africa

and

Department of Mathematics

University of Eswatini

Private Bag 4

Kwaluseni, Eswatini (Swaziland)

E-mail: sandilemotsa@gmail.com
Ramandeep Behl (corresponding author)

Department of Mathematics King Abdulaziz University Jeddah 21589, Saudi Arabia E-mail: ramanbehl87@yahoo.in 
